\title{
Dark Matter Determinations from Chandra Observations of Quadruply Lensed Quasars
}

\author{
David Pooley ${ }^{1}$, Jeffrey A. Blackburne ${ }^{2}$, Saul Rappaport ${ }^{2}$ \\ and Paul L. Schechter ${ }^{2}$ \\ ${ }^{1}$ Department of Astronomy, University of Wisconsin, Madison, WI 53706, USA \\ email: dave@astro.wisc.edu
}

${ }^{2}$ Kavli Institute for Astrophysics and Space Research, Massachusetts Institute of Technology, Cambridge, MA 02139, USA

We present Chandra X-ray observations of 14 quadruply lensed quasars. The X-ray data reveal flux ratio anomalies which are more extreme than those seen at optical wavelengths, confirming the microlensing origin of the anomalies originally seen in the optical data. The reduction of the anomalies in the optical, as compared to X-ray, indicates that the sizes of the optical emitting regions of the quasars must be about $1 / 3$ the size of the projected Einstein radii of the microlensing stars. The X-ray emitting regions are essentially point sources and therefore give a microlensing signal unencumbered by source size considerations. For each lensing galaxy, we determine the most likely ratio of smooth material (dark matter) to clumpy material (stars) to explain the X-ray flux ratios. The ensemble of Chandra-observed quads indicates that the amount of matter projected along the lines of sight to the images (at radial distances of several kpc from the centers of the lensing galaxies) is $\sim 90 \%$ smooth dark matter and $\sim 10 \%$ stars. 